\title{
Incidence of traumatic long-bone fractures requiring inhospital management: a prospective age- and gender-specific analysis in 4,890 fractures Terje Meling*1, Knut Harboe ${ }^{1}$ and Kjetil Søreide ${ }^{2,3}$
}

\author{
Address: ${ }^{1}$ Department of Orthopedic Surgery, Stavanger University Hospital, Stavanger, Norway, ${ }^{2}$ Department of Surgery, Stavanger University \\ Hospital, Stavanger, Norway and ${ }^{3}$ Department of Surgical Sciences, University of Bergen, Bergen, Norway \\ Email: Terje Meling* - terje_melling@yahoo.no \\ * Corresponding author
}

from Scandinavian Update on Trauma, Resuscitation and Emergency Medicine 2009

Stavanger, Norway. 23 - 25 April 2009

Published: 28 August 2009

Scandinavian Journal of Trauma, Resuscitation and Emergency Medicine 2009, I7(Suppl 3):OI5 doi:10.1 186/I757-724I-I7-S3-OI5

This abstract is available from: http://www.sjtrem.com/content/I7/S3/OI5

(C) 2009 Meling et al; licensee BioMed Central Ltd.

\section{Background}

Musculoskeletal trauma represents a considerable global health burden, however reliable population-based incidence data are lacking. Thus, we prospectively investigated the age- and sex-specific incidence patterns of longbone fractures in a defined population.

\section{Methods}

A 4-year prospective study of all long-bone fractures in a defined Norwegian population. Demographic data, fracture type and location, and mode of treatment were collected using recognized classification (e.g. AO/OTA; Gustilo-Anderson for open fractures). Age- and sexadjusted incidences were calculated using population statistics.

\section{Results}

During the study period 4,890 long-bone fractures were recorded. Overall incidence per 100,000/yr was 406 with $95 \%$ confidence interval $(95 \% \mathrm{CI})$ of $395-417$. The ageadjusted incidence $<16$ yrs $(339 ; 95 \% \mathrm{CI} 318-360)$ was lower than those $\geq 16$ years (427; 95\% CI: 414-440). Overall male incidence $(337 ; 95 \% \mathrm{CI} 322-355)$ was lower than female $(476 ; 95 \%$ CI $459-493)$, but male:female ratio was 2:1 among those $<50$ years, and 1:3 in those $\geq 50$ years. Upper limb fractures had an overall incidence of 159 (95\% CI 152-166), whereas lower limb incidence was 247 (95\%CI 238-256). Open fractures occurred in 3\%, for an incidence of 13 (95\%CI 11-15). Pediatric fractures were more often treated conservatively with only $8 \%$ requiring internal fixation, compared to $56 \%$ internal fixation in those $\geq 16$ years. An increase in the use of angular stable plates occurred during the study period.

\section{Conclusion}

This prospectively collected study of long-bone fractures in a defined population recognizes age- and gender-specific fracture patterns. Boys predominate in young age for which treatment is basically conservative. In the senior population, women and operative treatment predominate. 